\title{
Measuring Effectiveness of Glimepiride Titration Using SMBG in Patients with Mild Type 2 Diabetes
}

\author{
Akio Kanazawa ${ }^{*}, 1$, Tomoaki Shimizu ${ }^{1}$, Chie Ebato ${ }^{1}$, Yuko Sakurai ${ }^{1}$, Naoki Kumashiro ${ }^{1}$, Shinya Miwa ${ }^{1}$, \\ Takahisa Hirose ${ }^{1,2}$, Yasushi Tanaka ${ }^{4}$, Ryuzo Kawamori ${ }^{1,2,3}$ and Hirotaka Watada ${ }^{1}$ \\ ${ }^{1}$ Department of Medicine, Metabolism and Endocrinology, ${ }^{2}$ Center for Therapeutic Innovations in Diabetes, and \\ ${ }^{3}$ Center for Beta Cell Biology and Regeneration, Juntendo University School of Medicine, Tokyo, Japan \\ ${ }^{4}$ Department of Internal Medicine, Division of Metabolism and Endocrinology, St. Marianna University School of \\ Medicine, Kawasaki, Kanagawa, Japan
}

\begin{abstract}
Glimepiride is a potent sulfonylurea agent and is widely used for type 2 diabetic patients, however, the safety and efficacy of glimepiride in patients with fair diabetic control (HbA1c level: 6.5-7.9\%) have not been investigated so far. Therefore, we investigated the safety and efficacy of glimepiride titration using self-monitoring blood glucose (SMBG) in the achievement of strict glycemic control in fairly controlled diabetic patients. Japanese type 2 diabetic patients who were diet-controlled or treated with alpha-glucosidase inhibitor or metformin, were randomly assigned into the SMBG group with titration of glimepiride using SMBG, or the conventional therapy group (control group) without SMBG. Glimepiride was initiated at a dose of $0.5 \mathrm{mg} /$ day and plasma glucose, insulin, HbA1c, and glycoalbumin levels were evaluated for 6 months in both groups. The dose of glimepiride was titrated in the SMBG group according to the SMBG levels before breakfast and dinner. The mean dose of glimepiride at 6 months tended to be higher in the SMBG than the control group $(1.0 \pm 0.8$ vs $0.6 \pm 0.3 \mathrm{mg} /$ day $)$, but not significant. At 6 months after glimepiride treatment, HbA1c levels were significantly lower than at baseline (SMBG: $7.2 \pm 0.5$ vs $6.5 \pm 0.6 \%, \mathrm{n}=23, P<0.01$, control: $7.3 \pm 0.4$ vs $6.5 \pm 0.7 \%, \mathrm{n}=24, P<0.01)$, although they were similar at 6 months in the two groups. Only three hypoglycemic episodes were recorded among 50 subjects. We found no efficacy of glimepiride titration protocol in this study. However, glimepiride significantly improved glycemic control in fairly controlled diabetic patients without severe hypoglycemia.
\end{abstract}

Keywords: Sulfonylurea agent, SMBG, titration.

\section{INTRODUCTION}

The early stage of type 2 diabetes is characterized by loss of early insulin secretion from pancreatic $\beta$-cells, leading to postprandial hyperglycemia [1]. Postprandial hyperglycemia is an important factor in the progression of atherosclerosis $[2,3]$. Therefore, some drugs such as $\alpha$-glucosidase inhibitor and/or glinide, which control postprandial hyperglycemia, have been used as the first choice of anti-diabetic agents. Indeed, the relative contribution of postprandial glucose excursions to glycosylated hemoglobin (HbAlc) level is important in well-controlled patients, but the contribution of fasting hyperglycemia increases gradually with worsening of diabetes [4]. Therefore, when considering treatment of type 2 diabetic patients with moderately elevated $\mathrm{HbAlc}$ level (>7\%), high fasting blood glucose levels should be targeted also through careful management strategies.

Glimepiride is a potent sulfonylurea agent and is widely used for type 2 diabetic patients [5]. In addition to its effects on pancreatic B-cell function, glimepiride also enhances tissue sensitivity to insulin $[6,7]$ and has a favorable safety and efficacy profile with once-daily dosing [8]. Although the

*Address correspondence to this author at the Department of Medicine, Metabolism and Endocrinology, Juntendo University, School of Medicine, 2-1-1 Hongo, Bunkyo-ku, Tokyo 113-8421, Japan; Tel: 81-3-5802-1579; Fax: 81-3-3813-5996; E-mail: akana@juntendo.ac.jp safety and efficacy of glimepiride are well documented in poorly controlled diabetic patients with low insulin secretion $[9,10]$, the safety and efficacy of glimepiride in patients with fair diabetic control (HbA1c level: 6.5-7.9\%) have not been investigated so far. Furthermore, glimepiride treatment is well-known to have a low rate of hypoglycemia due to a weak stimulation of $\beta$-cells, but, when using glimepiride for patients with fair diabetic control, hypoglycemia may occur before meals, accompanying body weight gain. Therefore, glimepiride titration using SMBG could be useful in the achievement of strict glycemic control through careful check of hypoglycemia. This study examined the safety and efficacy of glimepiride titration using SMBG in fairly controlled diabetic patients to achieve strict glycemic control.

\section{MATERIALS AND METHODOLOGY}

\section{Study Subjects}

Type 2 diabetic patients who visited Juntendo University Hospital, International Goodwill Hospital, Chiba Nishi Hospital, and Kasuga Clinic were enrolled in this prospective study. There are no biases in the laboratory analysis because measurements of plasma glucose and HbAlc levels are standardized in Japan. The inclusion criteria were men and women between the ages of 30 and 80 years with type 2 diabetes controlled by diet alone or treated with $\alpha$-glucosidase inhibitor or metformin, with $\mathrm{HbA}_{1 \mathrm{c}}$ 
values of $\geq 6.5 \%$ but $<8 \%$ over several months before enrollment. The exclusion criteria included symptomatic coronary artery disease, cerebrovascular disease, renal failure, severe liver dysfunction, and heart failure. Individuals also were excluded if they had been treated with a thiazolidinedione, other sulfonylureas and insulin injections. The study protocol was approved by the ethics committee at Juntendo University Hospital, and written informed consent was obtained from each patient. Therefore, there were no ethical concerns in this study.

\section{Titration of Glimepiride Using SMBG}

The enrolled patients were divided at random into the SMBG group and the conventional (control) group by the envelope method. Patients in the SMBG group visited the hospital once a month and were instructed to measure blood glucose level at home using a glucose meter (Glucocard, Arkray, Inc, Japan) before breakfast and dinner for three days right before they visited the hospital. Glimepiride was initiated at a dose of $0.5 \mathrm{mg}$ orally once daily in the morning and titrated to reach and maintain target glycemic goals as shown in Table 1. The target SMBG level was below 120 $\mathrm{mg} / \mathrm{dL}$ before breakfast and dinner. A glimepiride dose of $1.0 \mathrm{mg}$ (SMBG level $\geq 130 \mathrm{mg} / \mathrm{dL}$ ) or $0.5 \mathrm{mg}$ (SMBG level $120-129 \mathrm{mg} / \mathrm{dL}$ ) was added in the morning at $1,2,3,4$, and 5 months up to a maximum dose of $6.0 \mathrm{mg}$. If the SMBG level before breakfast did not reach the target level despite reaching the target level before dinner, a dose of $0.5 \mathrm{mg}$ was added in the evening. During their monthly visit to the hospital, the patients also provided blood samples for measurement of HbA1c, glycoalbumin, postprandial blood glucose, and postprandial immunoreactive insulin (IRI). For the control group, each patient was treated with glimepiride at a dose which was empirically decided by each clinician without a specific protocol. Patients in this group did not undergo home monitoring of blood glucose with a glucose meter. Patients in both groups were followed by diabetes specialists. This study spanned over 6 months and the target HbA1c level was $<6.5 \%$ at the endpoint.

Table 1. Titration of Glimepiride Using SMBG Level

\begin{tabular}{|c|c|c|}
\hline \multicolumn{2}{|c|}{ Glucose Levels (mg/dL) } & \multirow{2}{*}{$\begin{array}{c}\text { Titration Step } \\
\text { (Additional Dose of Glimepiride) }\end{array}$} \\
\hline Breakfast & Dinner & $1.0 \mathrm{mg}$ at breakfast \\
\hline$\geq 130$ & $\geq 130$ & $0.5 \mathrm{mg}$ at dinner \\
\hline$\geq 130$ & $<130$ & $0.5 \mathrm{mg}$ at breakfast \\
\hline $120-130$ & $\geq 120$ & $0.5 \mathrm{mg}$ at dinner \\
\hline $120-130$ & $<120$ & $0.5 \mathrm{mg}$ at breakfast \\
\hline$<120$ & $\geq 120$ & none \\
\hline$<120$ & $<120$ & \\
\hline
\end{tabular}

\section{Evaluation of Safety}

Safety outcome was judged from the appearance of adverse events and hypoglycemic episodes as well as the results of standard laboratory parameters. Clinically relevant hypoglycemia was defined as serum glucose of $<60 \mathrm{mg} / \mathrm{dL}$ or episodes requiring oral carbohydrates. Severe hypoglycemia was defined as either serum blood glucose $\leq$
$40 \mathrm{mg} / \mathrm{dL}$ or episodes requiring intravenous glucose injection.

\section{Statistical Analysis}

Comparisons between two groups and between baseline and endpoint in each group were performed by the Mann-Whitney $U$ and the Wilcoxon signed rank tests, respectively. Data are expressed as mean $\pm \mathrm{SD}$. A P value < 0.05 was considered statistically significant.

\section{RESULTS}

\section{Clinical Characteristics}

A total of 50 subjects were randomized into the SMBG group $(n=24)$ and the control group $(n=26)$. Of these subjects, 24 subjects in the SMBG group and 24 subjects in the control group completed the study. The baseline characteristics of both groups are summarized in Table $\mathbf{2}$. Subjects in the two groups were matched for age, sex, duration of diabetes, body mass index (BMI), and the levels of HbA1c, glucose, and glycoalbumin.

Table 2. Clinical Characteristics at Baseline

\begin{tabular}{|l|c|c|c|}
\hline & SMBG Group & Control Group & $\boldsymbol{p}$ \\
\hline \hline Gender (Male/Female) & $15 / 9$ & $21 / 5$ & \\
\hline Duration of diabetes (years) & $5.5 \pm 4.9$ & $5.6 \pm 6.3$ & NS \\
\hline Age (years) & $60.8 \pm 8.6$ & $58.7 \pm 10.1$ & NS \\
\hline BMI $\left(\mathrm{kg} / \mathrm{m}^{2}\right)$ & $23.3 \pm 4.3$ & $23.9 \pm 4.3$ & NS \\
\hline HbA1c $(\%)$ & $7.2 \pm 0.5$ & $7.3 \pm 0.4$ & NS \\
\hline Glycoalbumin $(\%)$ & $19.2 \pm 3.2$ & $19.6 \pm 2.6$ & NS \\
\hline Glucose $(\mathrm{mg} / \mathrm{dL})$ & $152.7 \pm 36.3$ & $166.1 \pm 46.0$ & NS \\
\hline IRI $(\mu \mathrm{U} / \mathrm{mL})$ & $13.1 \pm 9.9$ & $14.1 \pm 16.9$ & NS \\
\hline Anti-diabetic drugs & & & \\
\hline$\alpha$-GI & 9 & 4 & \\
\hline metformin & 2 & 5 & \\
\hline
\end{tabular}

\section{Glycemic Control in the SMBG and the Control Groups}

As shown by Table 3 , titration of glimepiride was achieved in the SMBG group based on the titration method, and all patients needed the administration of glimepiride. The mean dose of glimepiride at 6 months for the SMBG group tended to be higher than that used by the control patients, but not significant $(1.0 \pm 0.8 v s 0.6 \pm 0.3 \mathrm{mg} / \mathrm{day})$. Fig. (1) shows that glimepiride treatment was associated with significant falls in HbA1c (SMBG; $7.2 \pm 0.5$ vs $6.5 \pm$ $0.6 \%, \mathrm{n}=23, P<0.01$, control; $7.3 \pm 0.4$ vs $6.5 \pm 0.7 \%, \mathrm{n}=24$, $P<0.01$ ) and glycoalbumin levels (SMBG; $18.7 \pm 3.0$ vs 17.0 $\pm 2.8 \%, \mathrm{n}=14, P<0.01$, control; $19.6 \pm 2.6$ vs $17.2 \pm 2.6 \%$, $\mathrm{n}=21, P<0.01)$ in both groups, however, there were no significant differences in $\mathrm{HbA} 1 \mathrm{c}$ and glycoalbumin levels at 6 months between the two groups. Fig. (2) also demonstrates that postprandial glucose levels in the control group were significantly lower after 6 months of glimepiride treatment compared with baseline $(161.4 \pm 43.8$ vs $142.4 \pm 37.6$ $\mathrm{mg} / \mathrm{dL}, \mathrm{n}=22, P<0.05$ ), and postprandial glucose levels in the SMBG group tended to fall, but not significant $(152.7 \pm 36.3$ 

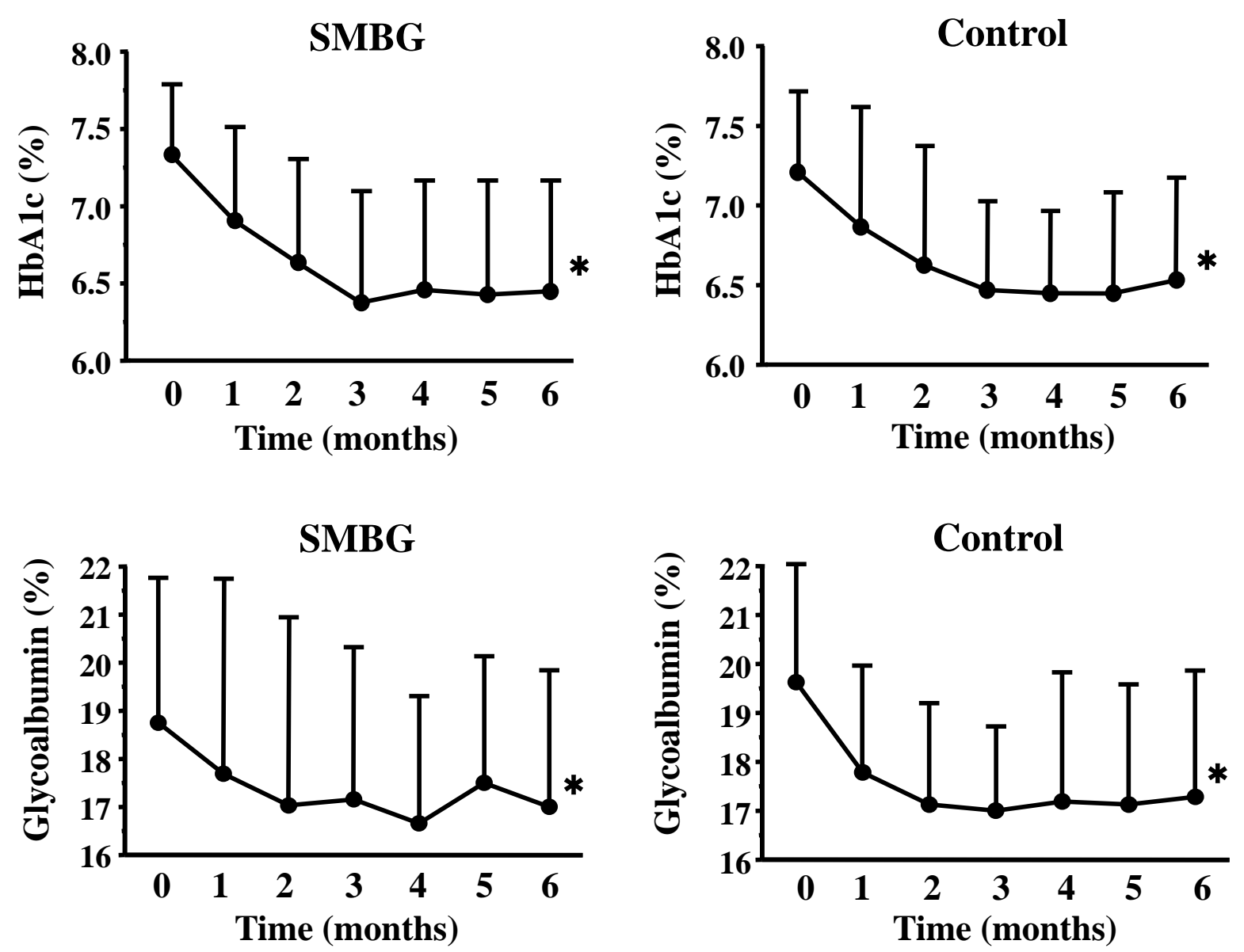

Fig. (1). Top panel: $\mathrm{HbA}_{1 \mathrm{c}}$ levels before and during 6-month treatment with glimepiride in the SMBG and the control groups. Bottom Panel: glycoalbumin levels before and during 6-month treatment with glimepiride in the SMBG and the control groups. Glimepiride significantly improved glycemic control in both groups. Data are mean \pm SD. $* P<0.01$, compared with the baseline.

vs $133.3 \pm 40.9 \mathrm{mg} / \mathrm{dL}, \mathrm{n}=23$, NS). Postprandial IRI levels in the control group were significantly higher at 6 months than at baseline $(10.8 \pm 7.3$ vs $26.7 \pm 24.6 \mu \mathrm{U} / \mathrm{mL}, P<0.01$, $\mathrm{n}=20)$, and postprandial IRI levels in the SMBG group showed a tendency to increase, but not significant $(12.8 \pm 9.1$ vs $22.3 \pm$ $20.1 \mu \mathrm{U} / \mathrm{mL}, \mathrm{n}=15$, NS). Fig. (3) presents that there was no significant difference in BMI before and after glimepiride treatment, indicating that these glimepiride-induced changes were not due to changes in BMI.

\section{Analysis of Glycemic Control Focused on a Dose of Glimepiride}

As shown by Table $\mathbf{3}$, because more than half of the patients in this study were treated with $0.5 \mathrm{mg} / \mathrm{day}$ of glimepiride, we reanalyzed glycemic control by dividing the entire group into two subgroups. The first is the $0.5 \mathrm{mg} /$ day group $(n=32)$, in which the initial dose of $0.5 \mathrm{mg} /$ day was persistently used throughout the study, while the second consisted of patients who required glimepiride at $>0.5$ $\mathrm{mg} /$ day $(\mathrm{n}=15)$. Fig. (4) shows the serial changes in HbA1c levels from baseline to 6 months for the two subgroups. Significant reductions in HbA1c levels at the endpoint were noted in both the $0.5 \mathrm{mg}$ group $(7.1 \pm 0.4$ vs $6.2 \pm 0.4 \%, \mathrm{n}=32$, $P<0.01)$ and $>0.5 \mathrm{mg}$ group $(7.5 \pm 0.4$ vs $7.0 \pm 0.8 \%, \mathrm{n}=15$, $P<0.01)$. Furthermore, the HbA1c level at 6 months was significantly lower in the $0.5 \mathrm{mg}$ group than the $>0.5 \mathrm{mg}$ group $(6.2 \pm 0.4 v s 7.0 \pm 0.8 \%, P<0.01)$. The mean IRI level was significantly higher after glimepiride treatment in the $0.5 \mathrm{mg}$ group $(13.2 \pm 9.0$ vs $29.9 \pm 25.3 \mu \mathrm{U} / \mathrm{ml}, P<0.01$, $\mathrm{n}=23)$, but not in the $>0.5 \mathrm{mg}$ group $(8.4 \pm 4.5 \mathrm{vs} 14.6 \pm 12.1$ $\mu \mathrm{U} / \mathrm{ml}$, NS, $\mathrm{n}=11)$, and there were no significant differences in clinical backgrounds before glimepiride treatment between the two groups (estimated duration of diabetes: 5.5 \pm 5.8 vs $5.9 \pm 5.8$ years, BMI: $22.9 \pm 3.8$ vs $23.8 \pm 2.2 \mathrm{~kg} / \mathrm{m}^{2}$, and age: $60.6 \pm 7.9 v s 60.2 \pm 11.9$ years, the $0.5 \mathrm{mg}$ group $v s$ the $>0.5 \mathrm{mg}$ group, NS) Additionally, there were no significant differences in the percentage of the prior medications ( $\alpha$-GI: 21.9 vs 40.0\%, metformin: 15.6 vs $13.3 \%$, none 62.5 vs $46.7 \%$, NS, the $0.5 \mathrm{mg}$ group $v s$ the $>0.5 \mathrm{mg}$ group).

\section{Safety}

The incidence of adverse events and episodes of hypoglycemia were evaluated in 50 subjects who were initially enrolled in this study. Hypoglycemic episodes occurred before meal in three patients (two patients in the control group and one in the SMBG group) who were treated with $0.5 \mathrm{mg} / \mathrm{day}$ glimepiride with an overall rate of hypoglycemia of $6.0 \%$. Treatment with glimepiride was discontinued in two patients in the control group due to the hypoglycemic episode, though it was not severe. No other adverse events occurred throughout the study. 

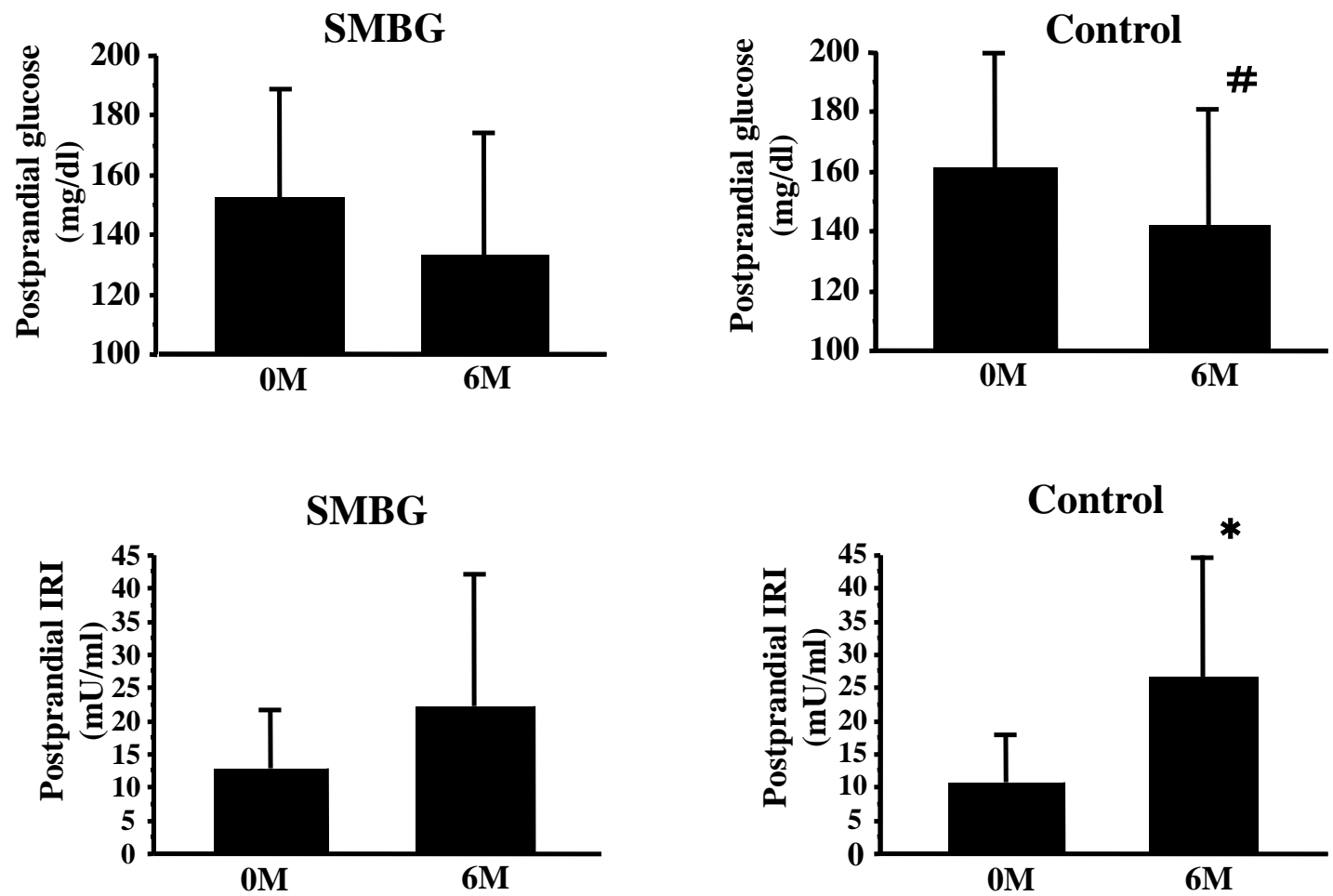

Fig. (2). Top panel: postprandial glucose levels before $(0 \mathrm{M})$ and after 6-month $(6 \mathrm{M})$ treatment with glimepiride in the SMBG and the control groups. Bottom Panel: postprandial IRI levels before $(0 \mathrm{M})$ and after 6-month $(6 \mathrm{M})$ treatment with glimepiride in the SMBG and the control groups. Data are mean $\pm \mathrm{SD}$. $* P<0.01$ and $\# P<0.05$, compared with the baseline.

SMBG

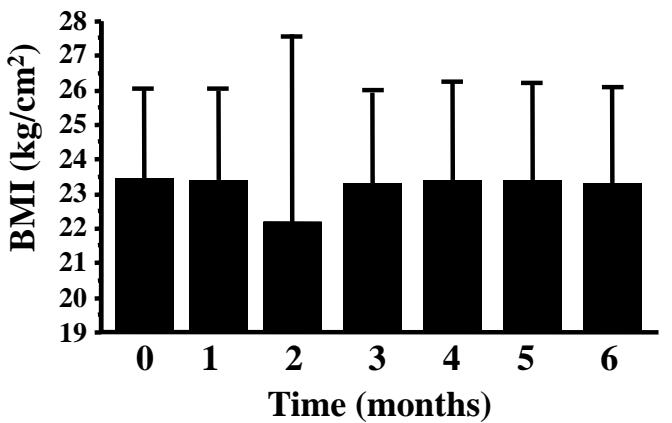

Control

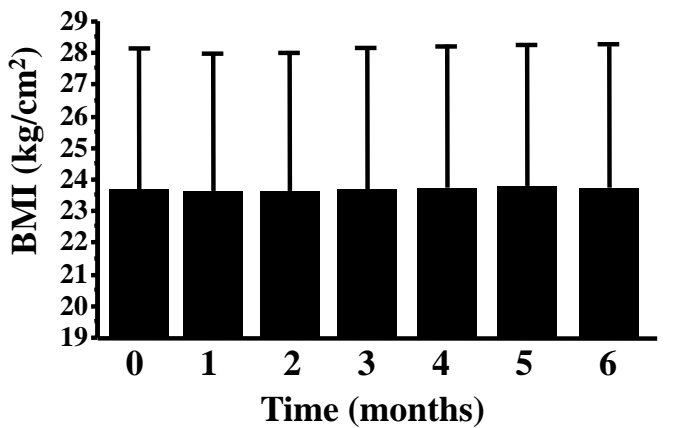

Fig. (3). Body mass index (BMI) before and during 6-month treatment with glimepiride in the SMBG and the control groups. Data are mean \pm SD.

Table 3. Dose of Glimepiride Used for the SMBG and Control Groups at 6 Months

\begin{tabular}{|c|c|c|}
\hline Glimepiride Dose & SMBG Group $(\mathbf{n}=\mathbf{2 4})$ & Control Group $(\mathbf{n}=\mathbf{2 4})$ \\
\hline \hline 0.5 & 13 & 19 \\
\hline 1.0 & 4 & 4 \\
\hline 1.5 & 2 & 0 \\
\hline 2.0 & 2 & 1 \\
\hline 2.5 & 1 & 0 \\
\hline 3.0 & 2 & 0 \\
\hline$\geq 3.5$ & 0 & 0 \\
\hline Mean \pm SD dose & $\mathbf{1 . 0} \pm \mathbf{0 . 8}$ & $\mathbf{0 . 6} \pm \mathbf{0 . 3}$ \\
\hline
\end{tabular}

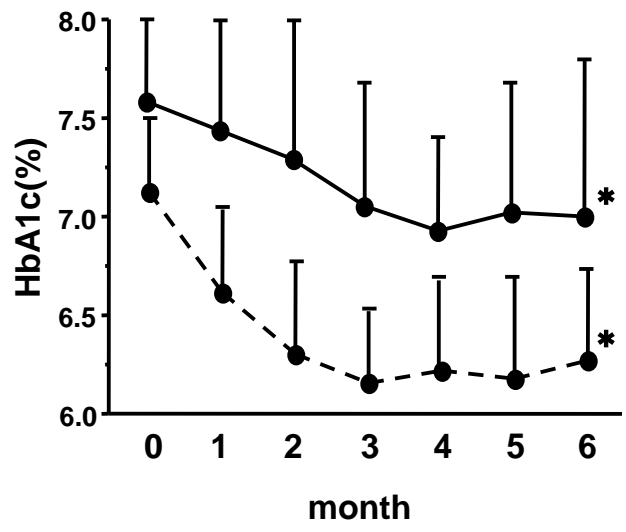

Fig. (4). $\mathrm{HbA}_{1 \mathrm{c}}$ levels before and during 6-month treatment with glimepiride at $0.5 \mathrm{mg} /$ day (dotted line) and $>0.5 \mathrm{mg} /$ day (solid line). ${ }^{*} P<0.01$, compared with the baseline. 


\section{DISCUSSION}

In this study, we examined the safety and efficacy of glimepiride titration using SMBG in fairly controlled diabetic patients to achieve strict glycemic control. To our knowledge, this is the first report on the titration of glimepiride using SMBG in diabetic patients. The results showed a significant improvement of glycemic control with glimepiride treatment in both the SMBG and the control groups, although the glycemic control and the dose of glimepiride at the endpoint in the two groups were not different. For these reasons, it was considered that 1) intensive titration in this study was not needed because more than half of the patients were treated with $0.5 \mathrm{mg}$ daily dose during the study period, and their glycemic control at the endpoint showed the target $\mathrm{HbA1c}$ levels below $6.5 \%$, and 2) our titration protocol needed to aim at the much lower SMBG levels and if so, mean dose of glimepiride in the SMBG group might show a significant increase, presenting better glycemic control compared with the control group. As shown by Fig. (4), the other patients treated with $>0.5 \mathrm{mg}$ glimepiride/day did not reach the target $\mathrm{HbAlc}$ levels in spite of the titration. In order to examine the ineffectiveness of titration, we examined the patient's background between the $0.5 \mathrm{mg}$ and the $>0.5 \mathrm{mg}$ groups. In both groups, there were no significant differences in age, BMI, estimated diabetic duration, prior diabetic medication. Therefore, the precise reason for the ineffectiveness of our titration protocol remains unknown. However, considering the finding that IRI levels in the $>0.5 \mathrm{mg}$ group were not higher compared with the $0.5 \mathrm{mg}$ group, it is possible that some patients are less sensitive to glimepiride stimulation. Additionally, it is very difficult to accurately measure diabetes duration in type 2 diabetes, and the other investigator has demonstrated that glucose-mediated insulin secretion inversely correlates with the duration of diabetes [11]. Therefore, we are unable to deny the possibility that inaccuracy of diabetes duration might affect, in part, the difference of sensitivity to glimepiride stimulation.

In the patients who were sensitive to glimepiride stimulation, glimepiride treatment resulted in increased postprandial levels of insulin first followed by reductions in postprandial glucose later, indicating that glimepiride lowered postprandial glucose by effectively stimulating pancreatic $\beta$-cells as well as decreased fasting glucose concentrations. However, some unfavorable effects should be considered regarding such sulfonylurea treatment. First, body weight gain due to the stimulatory effects to $\beta$-cells has been pointed out previously [12]. However, no body weight gain was noted during our study because glimepiride treatment has less likelihood of body weight gain with weak stimulatory effects on $\beta$-cells $[13,14]$. Second, hypoglycemia was reported previously [15]. In the present study of mild diabetics, only three patients developed mild hypoglycemic events before meals. However, this rate of hypoglycemia is not as high as that reported with glinides [16], and the SMBG group had fewer hypoglycemic events than the control group, suggesting that glucose monitoring before meals by SMBG might contribute to the fewer hypoglycemic events. Therefore, SMBG may be useful for check of hypoglycemia avoiding the excessive dose of glimepiride. Third, secondary failure of sulfonylurea is an important issue at present. As reported by the ADOPT [12] and CHICAGO studies [17], long-term treatment of sulfonylurea may cause $\beta$-cell dysfunction, resulting in aggravation of glycemic control [18, 19]. In this study, glycemic control improved rapidly as shown by the results of glycoalbumin and $\mathrm{HbA1c}$, which are comparable with those of the CHICAGO study. Although HbA1c levels begin to rise 4 months after glimepiride treatment in the CHICAGO study, deterioration of glycemic control was not found in our study. Overall mean dose of glimepiride at 6 months in this study was $0.84 \pm 0.66 \mathrm{mg} / \mathrm{day}$, which was lower than that of CHICAGO study. Therefore, low-dose glimepiride may not add an extra burden to $\beta$-cells, leading to stable glycemic control.

There have been many studies regarding the efficacy of SMBG in diabetic patients so far. However the results are controversial [20]. As this reason, uncontrolled bias and some problems in study design have been pointed out [21]. For example, the education level of the patients, the mode of treatment, duration of the trial, and study design (i.e. how the patients are taught to take action based on the SMBG results) could significantly influence the efficacy of SMBG. In the present study, main purpose of SMBG was titration of glimepiride, and patients were not instructed to have educational programs for intensification of diet and exercise therapies based on the results of SMBG. Therefore, we consider that beneficial effect of SMBG on glycemic control was weak.

\section{CONCLUSION}

We demonstrated in the present study that our glimepiride titration protocol based on SMBG was not useful in patients with mild diabetes (HbA1c <8\%) over the 6-month study period. In other words, it is concluded that titration protocol in our study was equal to the empirical titration by diabetes specialists. Additionally, we presented that glimepiride improved significantly glycemic control in patients with mild diabetes without severe hypoglycemia.

\section{ACKNOWLEDGEMENT}

The authors thank the co-medical staff at Juntendo University Hospital, International Goodwill Hospital, Chiba Nishi Hospital, and Kasuga Clinic.

\section{REFERENCES}

[1] Igarashi Y, Mita T, Mizuno R, et al. Characteristics of Japanese subjects who progress from normal to impaired glucose tolerance. Longitudinal study excluding changes in body weight. Endocr J 2005; 52: 683-9.

[2] Mita T, Watada H, Shimizu T, et al. Nateglinide reduces carotid intima-media thickening in type 2 diabetic patients under good glycemic control. Arterioscler Thromb Vasc Biol 2007; 27: 2456-62.

[3] Azuma K, Toyofuku Y, Iesaki T, et al. Acarbose, an alpha-glucosidase inhibitor, improves endothelial dysfunction in Goto-Kakizaki rats exhibiting repetitive blood glucose fluctuation. Biochem Biophys Res Commun 2006; 30: 688-93.

[4] Monnier L, Lapinski H, Colette C. Contributions of fasting and postprandial plasma glucose increments to the overall diurnal hyperglycemia of type 2 diabetic patients: variations with increasing levels of $\mathrm{HbA}(1 \mathrm{c})$. Diabetes Care 2003; 26: 881-5.

[5] Massi-Benedetti M. Glimepiride in type 2 diabetes mellitus: A review of the worldwide therapeutic experience. Clin Ther 2003; 25: 799-816.

[6] Clark HE, Matthews DR. The effect of glimepiride on pancreatic beta-cell function under hyperglycaemic clamp and 
hyperinsulinaemic, euglycaemic clamp conditions in non-insulin-dependent diabetes mellitus. Horm Metab Res 1996; 28: 445-50.

[7] Haupt A, Kausch C, Dahl D, et al. Effect of glimepiride on insulin-stimulated glycogen synthesis in cultured human skeletal muscle cells: a comparison to glibenclamide. Diabetes Care 2002; 25: 2129-32.

[8] Matsuki M, Matsuda M, Kohara K, et al. Pharmacokinetics and pharmacodynamics of glimepiride in type 2 diabetic patients: compared effects of once- $v s$ twice-daily dosing. Endocr J 2007; 54: 571-6.

[9] Ose H, Fukui M, Kitagawa Y, et al. Efficacy of glimepiride in patients with poorly controlled insulin-treated type 2 diabetes mellitus. Endocr J 2005; 52: 563-9.

[10] Goldberg RB, Holvey SM, Schneider J. A dose-response study of glimepiride in patients with NIDDM who have previously received sulfonylurea agents. The Glimepiride Protocol \#201 Study Group. Diabetes Care 1996; 19: 849-56.

[11] Kaku K, Kawasaki F, Kanda Y, et al. Retained capacity of glucose-mediated insulin secretion in patients with type 2 diabetes mellitus inversely correlates with the duration of diabetes. Diabetes Res Clin Pract 2004; 64: 221-3.

[12] Kahn SE, Haffner SM, Heise MA, et al. ADOPT Study Group. Glycemic durability of rosiglitazone, metformin, or glyburide monotherapy. N Engl J Med 2006; 355: 2427-43.

[13] Weitgasser R, Lechleitner M, Luger A, et al. Effects of glimepiride on $\mathrm{HbA}(1 \mathrm{c})$ and body weight in Type 2 diabetes: results of a 1.5-year follow-up study. Diabetes Res Clin Pract 2003; 61: 13-9.
[14] de Boer H, Jansen M, Koerts J, et al. Prevention of weight gain in type 2 diabetes requiring insulin treatment. Diabetes Obes Metab 2004; 6: 114-9.

[15] Amiel SA, Dixon T, Mann R, et al. Hypoglycaemia in Type 2 diabetes. Diabet Med 2008; 25: 245-54.

[16] Saloranta C, Hershon K, Ball M, et al. Efficacy and safety of nateglinide in type 2 diabetic patients with modest fasting hyperglycemia. J Clin Endocrinol Metab 2002; 87: 4171-6.

[17] Mazzone T, Meyer PM, Feinstein SB, et al. Effect of pioglitazone compared with glimepiride on carotid intima-media thickness in type 2 diabetes: a randomized trial. JAMA 2006; 296: 2572-81.

[18] Takahashi A, Nagashima K, Hamasaki A, et al. Sulfonylurea and glinide reduce insulin content, functional expression of K(ATP) channels, and accelerate apoptotic beta-cell death in the chronic phase. Diabetes Res Clin Pract 2007; 77: 343-50.

[19] Satoh J, Takahashi K, Takizawa Y, et al. Secondary sulfonylurea failure: comparison of period until insulin treatment between diabetic patients treated with gliclazide and glibenclamide. Diabetes Res Clin Pract 2005; 70: 291-7.

[20] Simon J, Gray A, Clarke P, et al. Diabetes Glycaemic Education and Monitoring Trial Group. Cost effectiveness of self monitoring of blood glucose in patients with non-insulin treated type 2 diabetes: economic evaluation of data from the DiGEM trial. BMJ 2008; 24 : 1177-80.

[21] Saudek CD, Derr RL, Kalyani RR Assessing glycemia in diabetes using self-monitoring blood glucose and hemoglobin A1c. JAMA 2006; 12: 1688-97.

(C) Kanazawa et al.; Licensee Bentham Open.

This is an open access article licensed under the terms of the Creative Commons Attribution Non-Commercial License (http://creativecommons.org/licenses/by-nc/ $3.0 /)$ which permits unrestricted, non-commercial use, distribution and reproduction in any medium, provided the work is properly cited. 